\title{
A NEW APPROACH TO THE ANALYTIC EVALUATION OF
} THERMONUCLEAR REACTION RATES

\author{
H.J. Haubold and R.W. John \\ Zentralinstitut für Astrophysik der \\ Akademie der Wissenschaften der DDR, \\ 15 Potsdam, German Democratic Republic
}

In our paper (Haubold and John 1978) we succeeded in a closed-form evaluation of the reaction rate by means of a special function, known as Meijer's G-function

$$
\begin{aligned}
& r_{i j}=\left(1-\frac{1}{2} \delta_{i j}\right) N_{i} N_{j}\left(\frac{8}{\pi^{2} \mu}\right)^{1 / 2}(k T)^{\mu-1 / 2} \frac{S^{(\mu)}(0)}{\mu !} G_{0,3}^{3,0}\left(\left.\left[\frac{x}{2}\right]^{2}\right|_{1+\mu, \frac{1}{2}, 0}\right), \\
& x=2 \pi\left(\frac{\mu}{2 k T}\right) 1 / 2 \frac{Z_{i} Z_{j} e^{2}}{\hbar} .
\end{aligned}
$$

This representation of the rate is appropriate to perform analytical operations (e.g. for the computation of energy generation in a fusion plasma).

Furthermore, from (1) easily follow approximate expressions for small and large values of the characteristic parameter Coulomb barrier energy divided by thermal energy, which appears in the argument of the G-function. For large values we get the asymptotic representation :

$$
\begin{aligned}
G_{0,3}^{3,0}\left(\left.\left[\frac{x}{2}\right]^{2}\right|_{1+\mu, \frac{1}{2}, 0}\right) \sim\left(\left[\frac{x}{2}\right]^{2}\right)^{\frac{2 \mu+1}{6}} \exp \left\{-3\left[\frac{x}{2}\right]^{2 / 3}\right\} \times \\
\quad \times\left(B_{0}(\mu)+B_{1}(\mu)\left[\frac{x}{2}\right]^{-2 / 3}+B_{2}(\mu)\left[\frac{x}{2}\right]^{-4 / 3}+\ldots\right)
\end{aligned}
$$

The G-function in (1) admits a convergent series expansion, which may be used for the approximate computation of the rate in the case of small values of the parameter:

$$
G_{0,3}^{3,0}\left(\left.\left[\frac{x}{2}\right]^{2}\right|_{1+\mu, \frac{1}{2}, 0}\right) \sim \sum_{n=0}^{\infty}\left(a_{n}(\mu) x^{n} \ln x+b_{n}(\mu) x^{n+g \mu}\right) .
$$

Haubold, H.J. and John, R.W.: 1978, "Astron. Nachr." 229, pp. 225-232; 1979, 300, pp. 173.

D. Sugimoto, D. Q. Lamb, and D. N. Schramm (eds.), Fundamental Problems in the Theory of Stellar Evolution, 317. Copyright $\odot 1981$ by the IAU. 